\title{
Research Article \\ Backup Hydrogen Maser Steering System for Galileo Precise Timing Facility
}

\author{
Qinghua Wang, Pascal Rochat, and Xavier Stehlin \\ SpectraTime, Vauseyon 29, 2000 Neuchâtel, Switzerland \\ Correspondence should be addressed to Qinghua Wang, qinghua@spectratime.com
}

Received 18 July 2007; Accepted 18 November 2007

Recommended by Demetrios Matsakis

Two hydrogen masers (HMs) are used in the Precise Timing Facility to provide the physical realization of Galileo System Time, insuring the extremely high short-term stability required for the navigation functions. In order to allow a smooth switch over between backup and primary HMs, the "backup HM steering algorithm" is developed. This acquires the phase difference measured between two HMs, computes a steering correction, and generates the steering correction to the backup HM via a PicoStepper with a 0.1 -picosecond resolution. The algorithm design is based on outlier removal and a proportional-integral filtering controller. To verify the steering operability and the loop performance, the overall backup HM steering system is simulated using real HM-HM measurements, and with simulated anomalies (phase/frequency spikes, jumps, and drift).

Copyright (c) 2008 Qinghua Wang et al. This is an open access article distributed under the Creative Commons Attribution License, which permits unrestricted use, distribution, and reproduction in any medium, provided the original work is properly cited.

\section{INTRODUCTION}

The Precise Timing Facility (PTF) is one of the key facilities of the Galileo ground segment. Its scope is to provide an accurate, stable, and precise Galileo System Time Master Clock (GST(MC)) to the Orbitography and Synchronization Processing Facility and to the other Galileo Control Center facilities.

Two PTFs are currently under development by two separate teams in Germany and Italy. The discussion provided in this paper refers to the Italian development [1], coordinated by the Consorzio Torino Time (CTT) in Torino, Italy, with the partnership and support of SpectraTime (former Temex Time) and T4Science in Neuchâtel, Switzerland, and Astrogeodynamic Observatory, Poland.

Two active hydrogen masers (a primary HM1 and a backup HM2) externally steered via a precision PicoStepper, provide the physical realization of GST(MC), insuring the extremely high short-term stability required for the navigation functions, in particular, to perform a reliable satellite clock modeling.

The "backup HM steering algorithm" is implemented in order to allow a smooth switch over between backup and primary HM in case of failure of the latter, without producing any significant effect in the GST continuity, uniformity, or short-term frequency stability. The algorithm acquires the phase difference between two HMs measured by a multichannel phase comparator (MCPC), and generates a steering correction to be applied to the backup HM via a PicoStepper with a 0.1 -picosecond resolution.

\section{ARCHITECTURE}

Figure 1 shows the architecture of the backup HM steering system, consisting of an MCPC, PicoSteppers (one per HM), and the algorithm.

In the nominal situation, PicoStepper1 applies the steering correction from PTF GST algorithm to steer the primary HM1 with "GST running" (GSTR) obtaining the GST(MC1). The phases of the two PicoSteppers' outputs are compared by MCPC, whose output is used by the "backup HM steering algorithm" to obtain the steering command to PicoStepper2 whose input is the backup HM2. Thus, the steered output of HM2 is kept in phase with HM1.

In case of the HM1 failure, the hot backup HM2 becomes the primary one by the PTF switching matrix. The previous phase offset "HM2(steered)-HM1" provides the seamless switch-over signal via PicoStepper2 which is now applied by the GSTR correction for GST(MC). 


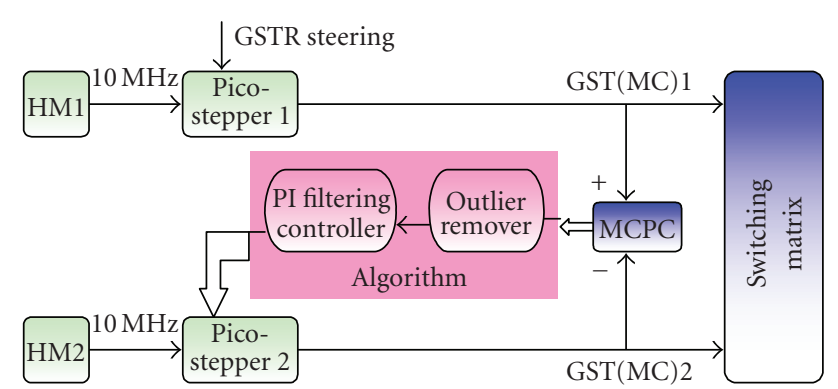

FIGURE 1: Architecture of the backup HM steering system.

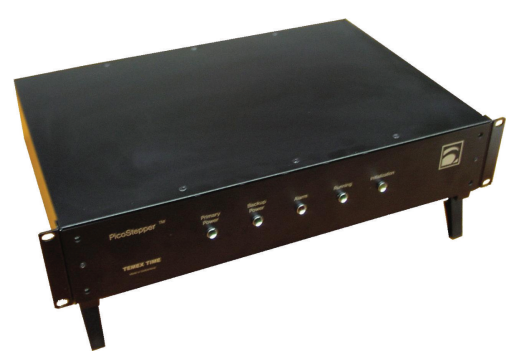

(a)

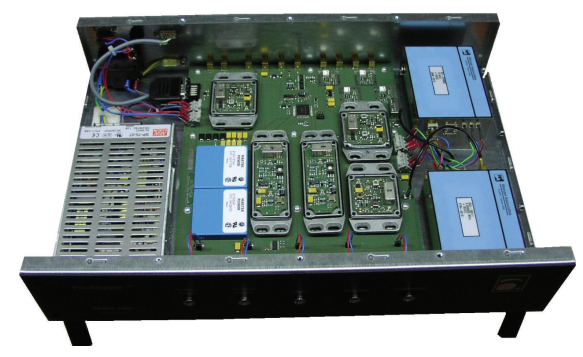

(b)

Figure 2: PTF PicoStepper.

\section{PICOSTEPPER}

A high-resolution PTF PicoStepper (i.e., microphase stepper), based on the existing PicoStepper by SpectraTime [2], is being developed to provide frequency correction of $\mathrm{HMs}$ signals (Figure 2).

The unit is being designed to meet the following two PTF requirements:

(i) increase of the resolution by a factor of 100 to obtain a minimum phase step of \pm 0.1 picosecond;

(ii) reduction of output jitter to get negligible degradation of the HM signal phase noise and short term stability.

The design is based on a double heterodyne architecture where a first structure is used for positive phase/frequency adjustment and the second structure for negative adjustment.

As shown in the high-level block diagram (Figure 3), each positive/negative loop contains a voltage-controlled xtal oscillator (VCXO), a phase detector, a frequency mixer, a frequency multiplier, a pulse removing circuit, a frequency di- vider and a loop filter. A microcontroller is in charge to manage the stepping commands sent by RS232. It has also the capability to execute a self-test of the unit.

The 0.1-picosecond resolution of the system is obtained by using the appropriate VCXO frequency multiplication factor and divider ratio. Taking $N=10$ and $M=10^{5}$, the frequency resolution is $\Delta f / f_{I N}=10^{-6} / 10 M= \pm 10^{-13}$, which corresponds in terms of phase of 0.1 picosecond.

The frequency beats (F1, F2, F3, and F4) in both loops while not stepping is equal to $1 \mathrm{KHz}$ which is the comparison frequencies of the phase detectors. Thus, the nominal frequency of the local oscillator is equal to $10 \mathrm{MHz}-1 \mathrm{KHz} / \mathrm{N}=$ $9.999900 \mathrm{MHz}$.

In order not to degrade the HM performances, a phase noise figure (Figure 4) analysis has been performed. The comparison between the HM specification and the best performances VCXO available on the market in terms of phase noise close to the carrier gives the required cutoff frequency to be implemented. It shows that the optimum cutoff frequency should be around $4 \mathrm{~Hz}$. Since the frequency beats used as comparison signal are $1 \mathrm{kHz}$, it is easy to implement the desired $4 \mathrm{~Hz}$ cutoff frequency.

\section{STEERING APPROACH}

The backup HM steering algorithm together with the MCPC and PicoStepper form a basic phase-locked loop (PLL), which locks the phase of the backup HM to the primary one. Figure 5 shows the block diagram of the backup HM steering model.

The algorithm design is based on a digital proportional integral (PI) filtering controller, which contains the PI filter and periodical generation of the steering commands accepted by the PicoStepper.

To eliminate the impact of anomalies of the primary HM output signal (e.g., phase spikes) on the steered backup HM, the algorithm first removes the phase outliers of the dynamic least-square linear fit (LSLF). Because the outlier routine is sensitive only to the difference between the two HMs, the steering computation is equally efficient at rejecting phase outliers from both the primary and the backup HMs. These outliers in the backup HM, however, remain in the steered output.

\subsection{Phase-locked loop and PI filter}

Figure 6 illustrates the PLL control system block diagram in the continuous (Laplace) domain.

The $s$-transfer function of second-order closed loop is

$$
C(s)=\frac{2 \xi \tau s+1}{\tau^{2} s^{2}+2 \xi \tau s+1}
$$

where $\tau$ is the loop time constant (in seconds), 1000 seconds, which is selected as the tradeoff of the time offset and the frequency stability [2]; $\xi$ is the damping factor, $1 ; K_{c}$ is MCPC gain, $10^{13} \mathrm{step} / \mathrm{s}$; and $K_{m}$ is the PicoStepper gain, $10^{-13} / \mathrm{step}$. 


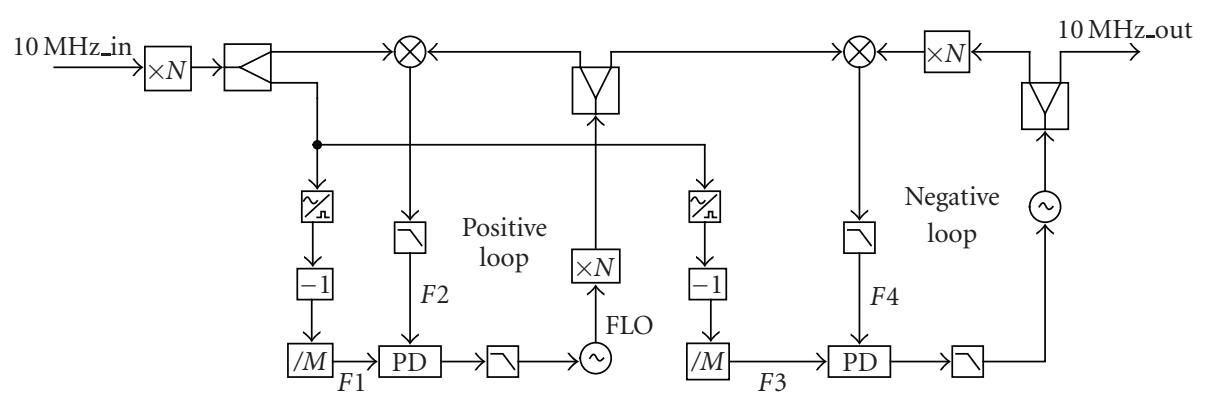

FIGURE 3: Block diagram of PTF PicoStepper (i.e., microphase stepper).

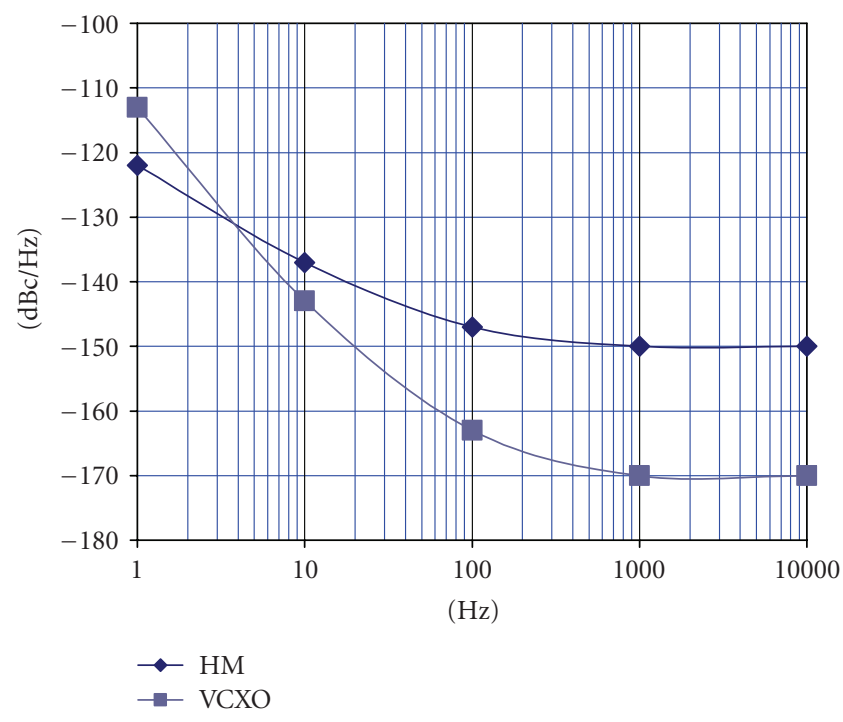

Figure 4: Phase noise figure.

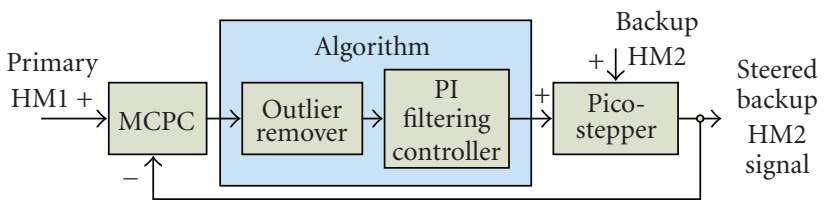

FIGURE 5: Block diagram of the backup HM steering model.

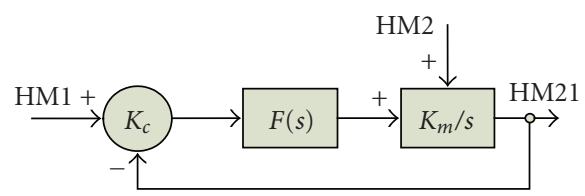

FIGURE 6: Block diagram of the phase-locked loop.

In discrete domain, basic digital filtering functions can be used. Therefore, the $z$-transfer function of the discrete PI filter is

$$
D(z)=K_{p}+K_{i} \frac{z}{z-1},
$$

where $K_{i}$ and $K_{p}$ are coefficients of the discrete integrator and proportional regulator.

\subsection{Dynamic least-square linear fitting and outlier removing}

Figure 7 illustrates the block diagram of the Outlier Remover. The input data from MCPC, $\mathrm{e} 0$ is checked by LSLF over the previous 100-second data in sliding windows. If the absolute value of the deviation from the fitting line exceeds the outlier criterion C (30 picoseconds), the data are removed and replaced by the previous value. Therefore, the phase outliers of the primary HM are filtered before the steering.

\section{BACKUP HM STEERING SYSTEM SIMULATION AND PERFORMANCE VERIFICATION}

The technical requirement on the backup HM steering system imposed by PTF design is that the phase jump will not exceed 30 picoseconds in the value of the GST(MC) to switch the primary and backup HMs.

A simulation model [3] is created to analyze and verify the steering operability and the loop performance under various test cases including the nominal and degraded conditions with simulated anomalies or feared events (phase/frequency spikes, jumps and drift) occurred in both HMs.

Figures 8, 9, 10, and 11 demonstrate the simulation results on various test cases. The backup HM2 is steered properly to the primary HM1 under all test cases.

(i) With phase spikes at the primary HM1, the algorithm properly eliminates the anomalies. The peak value of the phase offset "HM2(steered)-HM1(outliers removed)" is 4 picoseconds, which depends on the initial phase difference, and the standard deviation is 1.03 picoseconds after the loop is settled down.

(ii) In the presence of the phase step of 30 picoseconds (GST(MC) maximum phase jump) either at the primary HM1 or the backup HM2, the maximum impacted phase offset "HM2(steered)-HM1" is 8 picoseconds.

(iii) When the HM signal is applied by GST(MC) maximum frequency correction of 1e-14, the impact on the phase offset "HM2(steered)-HM1" is 6.3 picoseconds. 


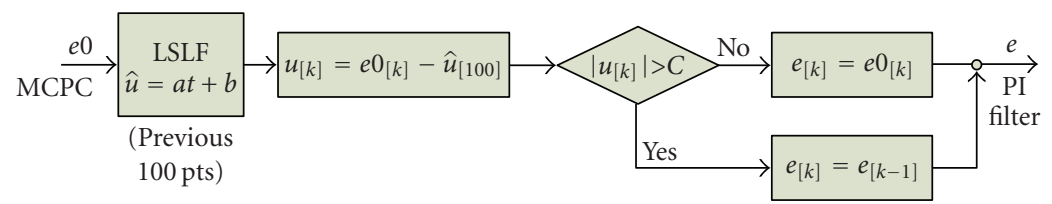

FIGURE 7: Block diagram of the outlier remover.

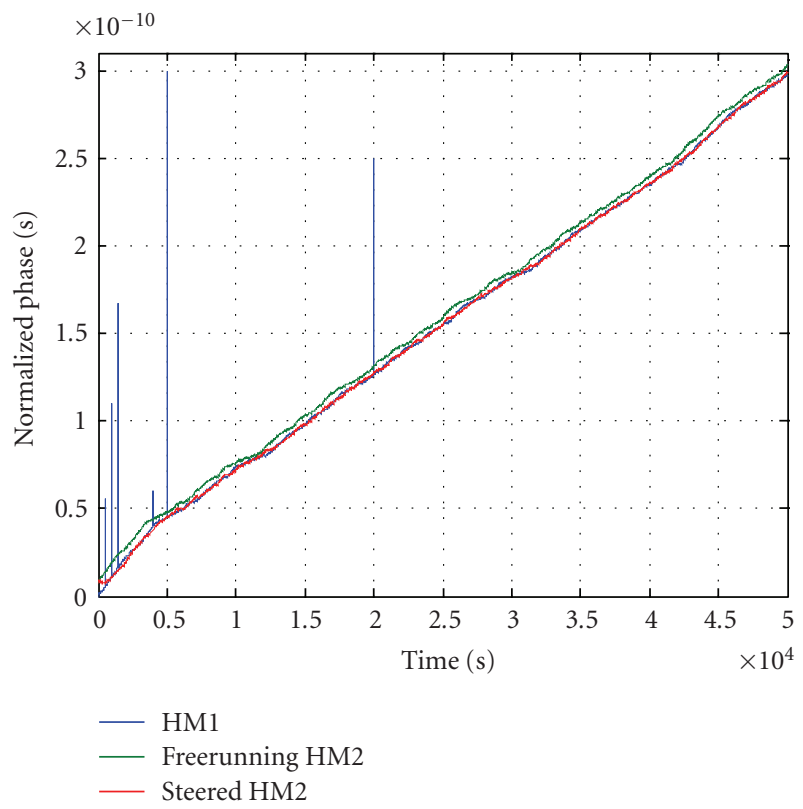

(a)

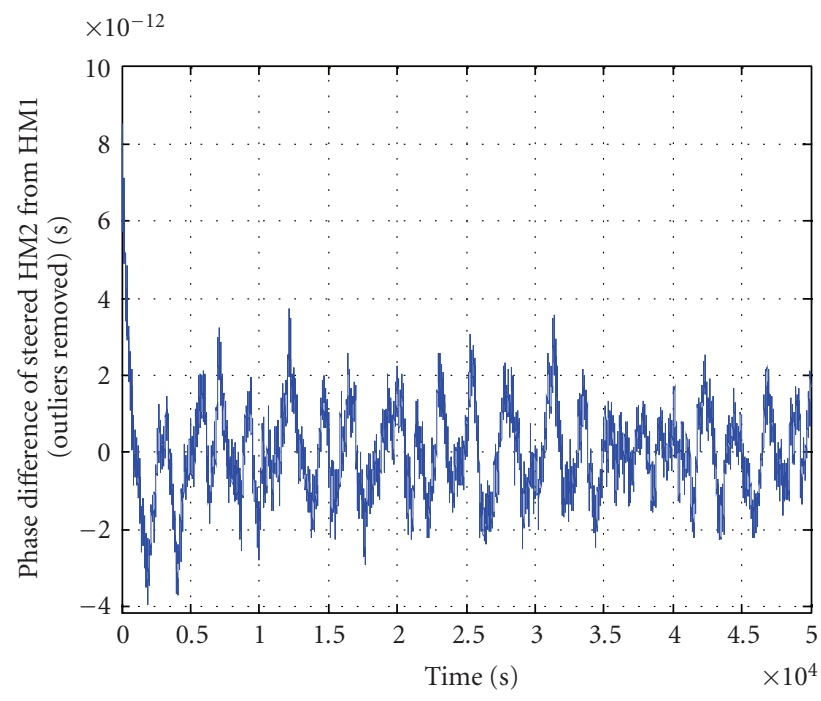

(b)

Figure 8: Simulation on phase/frequency spikes at primary HM1.

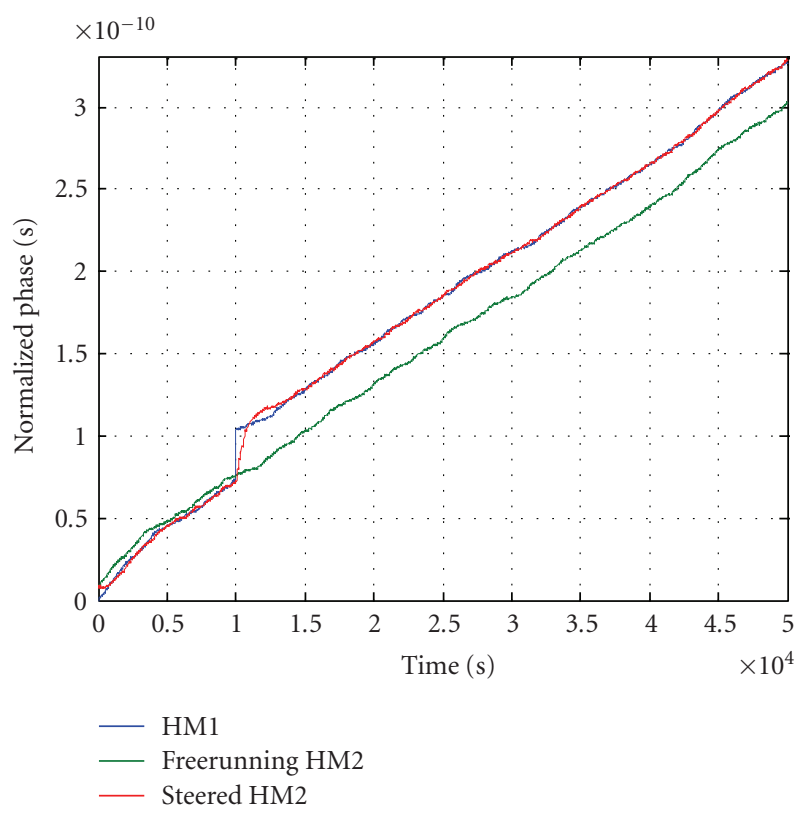

(a)

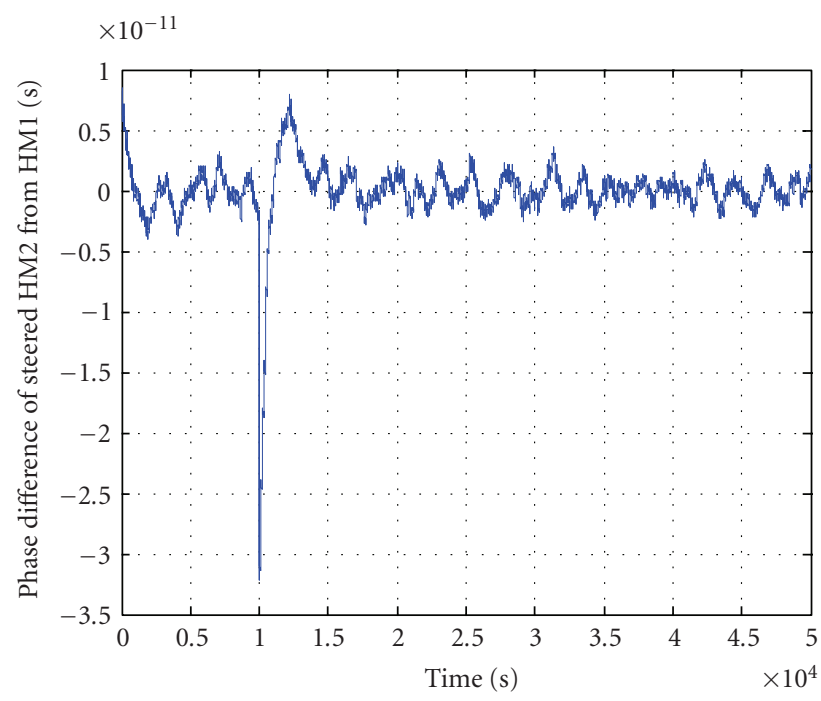

(b)

Figure 9: Simulation on phase jump of 30 picoseconds at HM1 (similar at HM2). 


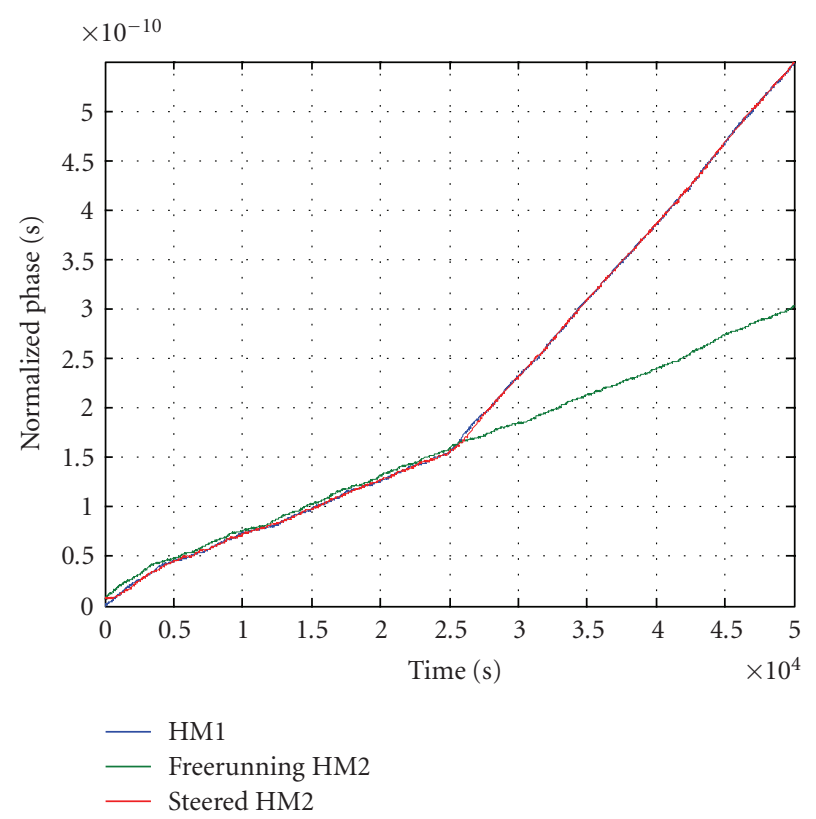

(a)

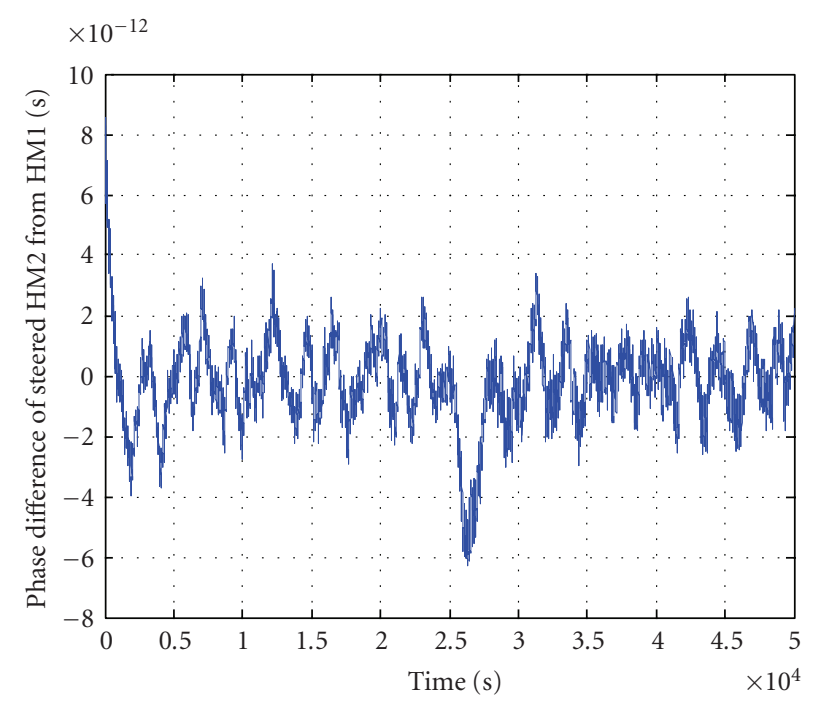

(b)

FIGURE 10: Simulation on frequency jump of 1e-14 at HM1 (similar at HM2).

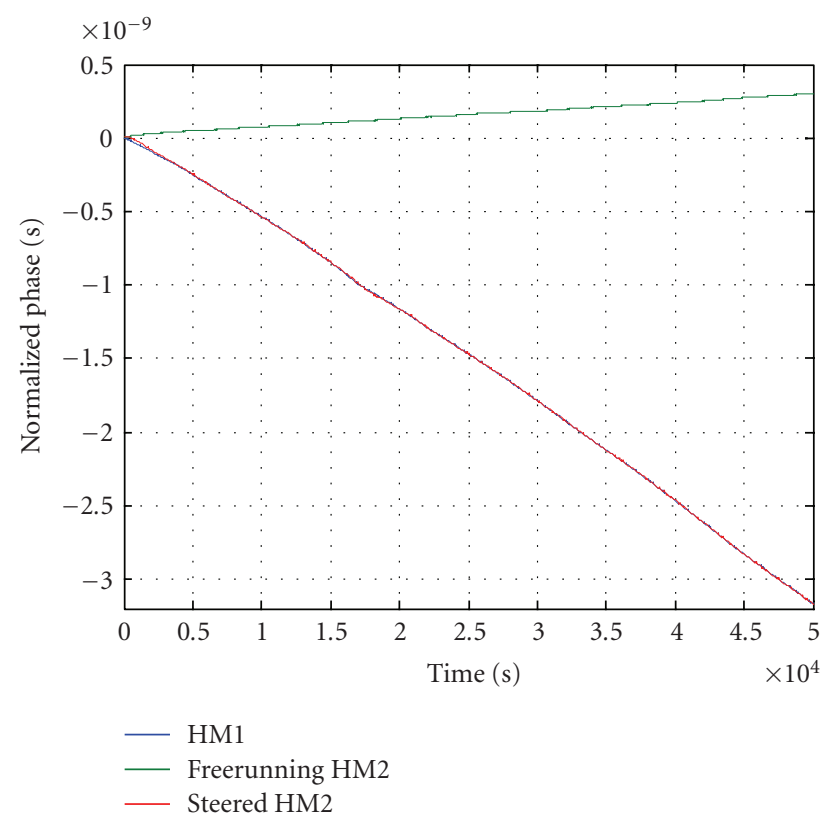

(a)

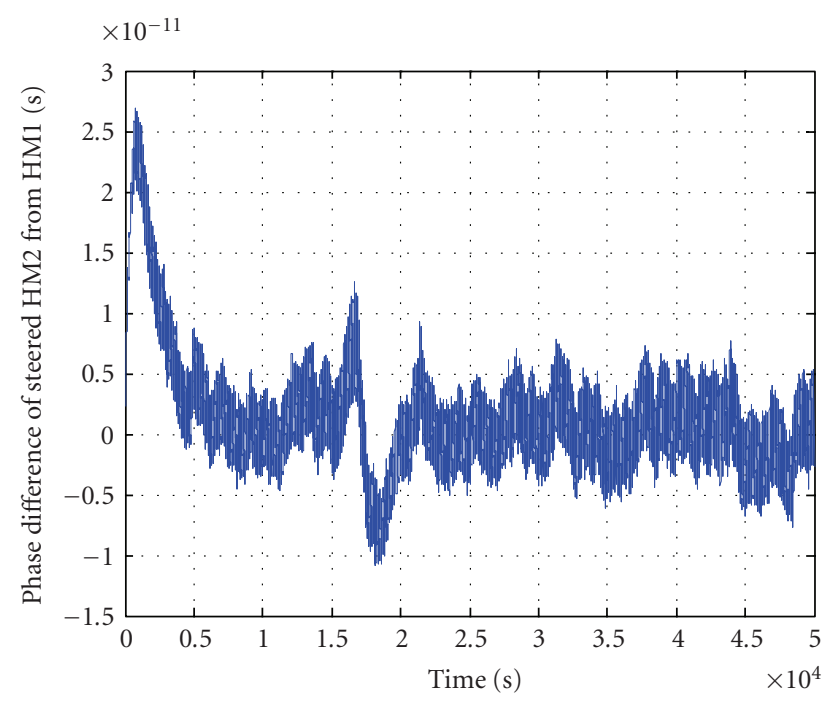

(b)

FIgURe 11: Simulation on frequency drift of 1e-13 at HM1 (similar at HM2).

(iv) Even if the HM frequency drift is seriously degraded, the phase offset "HM2(steered)-HM1" returns to specifications with the loop settling time, and the peak offset around the loop time constant is 27 picoseconds for the frequency drift of 1e-13/d (10 times worse of the specification of T4science HM, whose typical value is few e-15/d). The maximum phase offset as 12.5 picoseconds observed near to 20000 seconds is due to the accompanying frequency jump of $2.5 \mathrm{e}-14$ in the HM output signal. 
TABLE 1: Overall performance budget.

\begin{tabular}{|c|c|c|c|c|c|c|}
\hline \multirow{3}{*}{$\begin{array}{l}\text { Algorithm } \\
\text { simulation }\end{array}$} & Test case & 1 & 2 & 3 & 4 & 5 \\
\hline & Test event & Nominal & $\begin{array}{c}\text { Phase/frequency } \\
\text { spikes }\end{array}$ & $\begin{array}{c}\text { Phase jump of } 30 \mathrm{ps} \\
(G S T(M C) \\
\text { maximum } \\
\text { phase jump) } \\
\end{array}$ & $\begin{array}{l}\text { Frequency jump of 1e-14 } \\
\text { (GST(MC) maximum } \\
\text { frequency correction) }\end{array}$ & $\begin{array}{l}\text { Frequency drift of } \\
1 \mathrm{e}-13 / d \\
\text { (10 times worse of } \\
\text { HM specification) }\end{array}$ \\
\hline & Peak phase offset & $4 \mathrm{ps}$ & $4 \mathrm{ps}$ & $8 \mathrm{ps}$ & $6.3 \mathrm{ps}$ & $27 \mathrm{ps}$ \\
\hline \multicolumn{2}{|c|}{ Calibration accuracy } & \multicolumn{5}{|c|}{$5 \mathrm{ps}$} \\
\hline \multicolumn{2}{|c|}{ MCPC resolution } & \multicolumn{5}{|c|}{$0.1 \mathrm{ps}$} \\
\hline \multicolumn{2}{|c|}{ PicoStepper resolution } & \multicolumn{5}{|c|}{$0.1 \mathrm{ps}$} \\
\hline \multicolumn{2}{|c|}{ Total (phase offset) } & $6.4 \mathrm{ps}$ & $6.4 \mathrm{ps}$ & $9.4 \mathrm{ps}$ & $8 \mathrm{ps}$ & $27.5 \mathrm{ps}$ \\
\hline
\end{tabular}

Table 1 summarizes the overall performance budget, taking into account the calibration errors of the cables between the input of the MCPC and the input of the switching matrix, the MCPS resolution, and the PicoStepper resolution. The total performance is within the PTF requirement on the switch over phase jump of 30 picoseconds.

Besides above phase offset analysis, the frequency offset of "HM2(steered)-HM1" has been also calculated for all test cases, and it meets the PTF requirement that the frequency jump shall not exceed $4 \mathrm{e}-15$ over the averaging time of 100 minutes in the value of the GST(MC).

In addition, the worst cases are analysed.

(i) The PLL will be beyond the PicoStepper maximum control range $(1 e+4$ steps $)$ when the phase jump is bigger than 5 nanoseconds, or the frequency jump is bigger than $8 \mathrm{e}-12$.

(ii) For above latter case, the phase offset "HM2steeredHM1" is out of the specification of 30 picoseconds. To meet this specificaion, the frequency jump is allowed to be less than $5 \mathrm{e}-13$.

\section{CONCLUSION}

We conclude that our steering system is capable of meeting the Galileo specifications for keeping the backup HM close to the primary in phase and frequency. Currently the algorithm is in the detailed design phase and is passing a prototype phase subject to Galileo Software Standards. It will be tested on PTF hardwares, and will be implemented into the PTF operational software.

\section{REFERENCES}

[1] R. Zanello, M. Mascarello, L. Galleani, P. Tavella, E. Detoma, and A. Bellotti, "The Galileo precise timing facility," in Joint Meeting of the 21st European Frequency \& Time Forum and the IEEE International Frequency Control Symposium, pp. 458-462, Geneva, Switzerland, June 2007.

[2] X. Stehlin, Q. Wang, F. Jeanneret, P. Rochat, and E. Detoma, "Galileo system time physical generation," in Proceedings of the 38th Annual Precise Time and Time Interval (PTTI '06) Meeting, pp. 395-406, Reston, Va, USA, December 2006.
[3] Q. Wang and P. Rochat, "Backup hydrogen maser steering algorithm for Galileo precise timing facility," in Joint Meeting of the 21st European Frequency \& Time Forum and the IEEE International Frequency Control Symposium, pp. 463-466, Geneva, Switzerland, June 2007. 

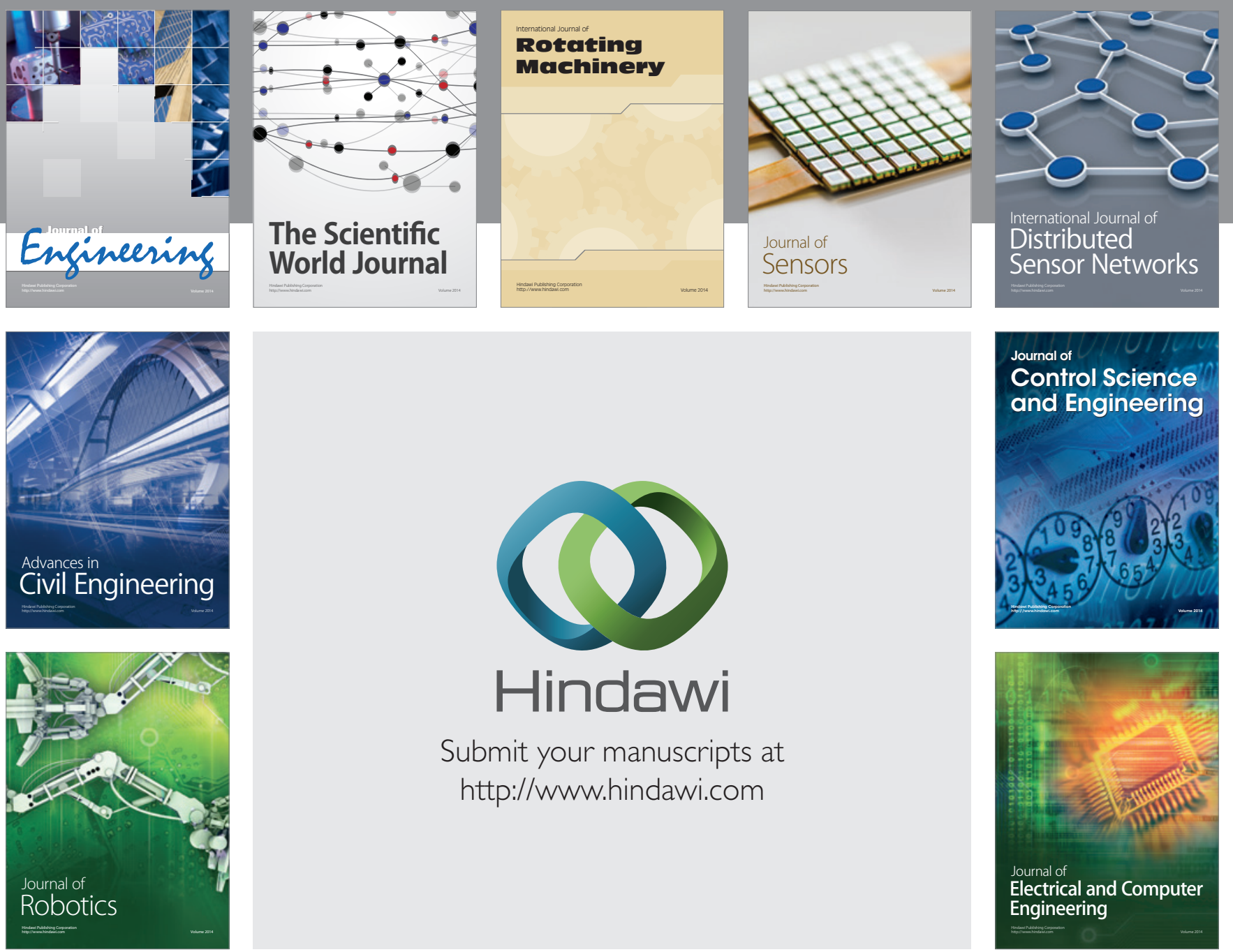

Submit your manuscripts at

http://www.hindawi.com
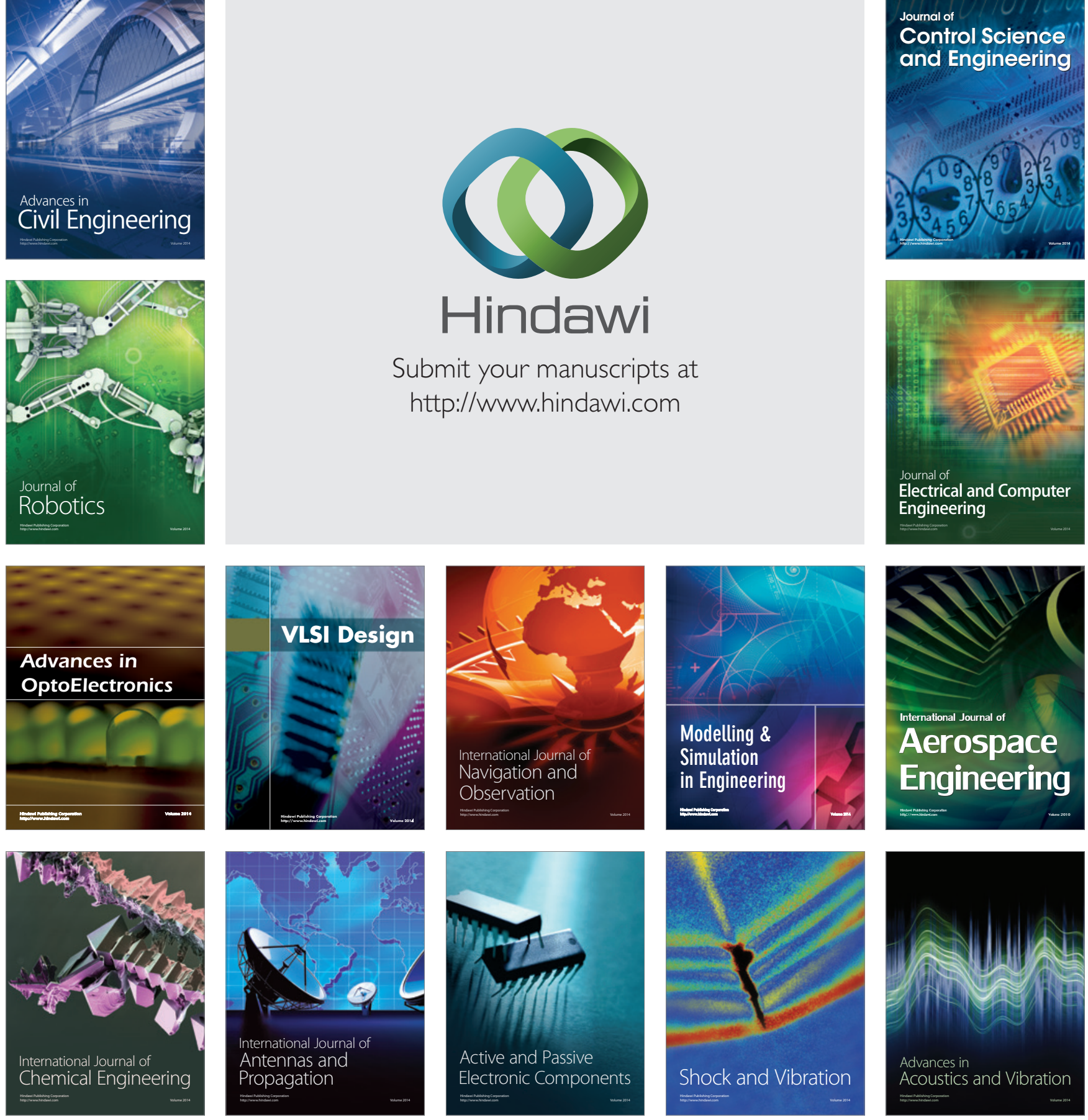